\title{
Insights from quantitative metaproteomics and protein-stable isotope probing into microbial ecology
}

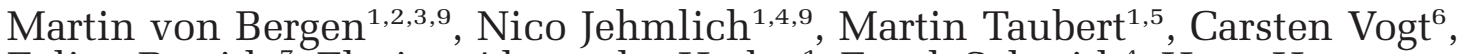 \\ Felipe Bastida $^{7}$, Florian-Alexander Herbst ${ }^{1}$, Frank Schmidt ${ }^{4}$, Hans-Hermann Richnow ${ }^{6}$ and \\ Jana Seifert ${ }^{1,8}$ \\ ${ }^{1}$ Department of Proteomics, UFZ_-Helmholtz Centre for Environmental Research, Leipzig, Germany; \\ ${ }^{2}$ Department of Metabolomics, UFZ-Helmholtz Centre for Environmental Research, Leipzig, Germany; \\ ${ }^{3}$ Department of Biotechnology, Chemistry and Environmental Engineering, Aalborg University, \\ Aalborg, Denmark; ${ }^{4}$ Interfaculty Institute for Genetics and Functional Genomics, University of Greifswald, \\ Greifswald, Germany; ${ }^{5}$ School of Environmental Sciences, University of East Anglia, Norwich, UK; \\ ${ }^{6}$ Department of Isotope Biogeochemistry, UFZ-Helmholtz Centre for Environmental Research, Leipzig, \\ Germany; ${ }^{7}$ Department of Soil and Water Conservation, Group of Soil Enzymology and Bioremediation and \\ Organic Wastes CEBAS-CSIC, Campus Universitario de Espinardo, Espinardo, Murcia, Spain and \\ ${ }^{8}$ Institute of Animal Nutrition, University of Hohenheim, Stuttgart, Germany
}

\begin{abstract}
The recent development of metaproteomics has enabled the direct identification and quantification of expressed proteins from microbial communities in situ, without the need for microbial enrichment. This became possible by (1) significant increases in quality and quantity of metagenome data and by improvements of (2) accuracy and (3) sensitivity of modern mass spectrometers (MS). The identification of physiologically relevant enzymes can help to understand the role of specific species within a community or an ecological niche. Beside identification, relative and absolute quantitation is also crucial. We will review label-free and label-based methods of quantitation in MS-based proteome analysis and the contribution of quantitative proteome data to microbial ecology. Additionally, approaches of protein-based stable isotope probing (protein-SIP) for deciphering community structures are reviewed. Information on the species-specific metabolic activity can be obtained when substrates or nutrients are labeled with stable isotopes in a proteinSIP approach. The stable isotopes $\left({ }^{13} \mathrm{C},{ }^{15} \mathrm{~N},{ }^{36} \mathrm{~S}\right)$ are incorporated into proteins and the rate of incorporation can be used for assessing the metabolic activity of the corresponding species. We will focus on the relevance of the metabolic and phylogenetic information retrieved with protein-SIP studies and for detecting and quantifying the carbon flux within microbial consortia. Furthermore, the combination of protein-SIP with established tools in microbial ecology such as other stable isotope probing techniques are discussed.
\end{abstract}

The ISME Journal (2013) 7, 1877-1885; doi:10.1038/ismej.2013.78; published online 16 May 2013

Subject Category: Integrated genomics and post-genomics approaches in microbial ecology

Keywords: quantitative metaproteomics; label-free quantification; spectral counting; stable isotope probing; protein-SIP; functional microbial community

\section{Introduction}

Contemporary microbial ecology studies use one or several 'omics' approaches to gain a deeper insight into the structure and function of microbial communities. Among these approaches, metaproteomics provides the direct link between genetic potential

Correspondence: J Seifert, Department of Proteomics, UFZHelmholtz Centre for Environmental Research, Permoserstrasse 15, D-04318 Leipzig, Germany.

E-mail: jana.seifert@ufz.de

${ }^{9}$ These authors contributed equally to this work.

Received 12 November 2012; revised 8 April 2013; accepted 13

April 2013; published online 16 May 2013 and actual functionality of the community. In recent years, a number of authors reviewed the steadily increasing number of metaproteomic studies and the ongoing technical progress, and almost all concluded that there is a need for quantitative input of metaproteomic studies (Keller and Hettich, 2009; Wilmes and Bond, 2009; Verberkmoes et al., 2009a; Schneider and Riedel, 2010). The rationale behind this is the often found redundancy of functionalities in complex communities, requiring quantitative data to decide which species is mainly responsible for some metabolic functions. Quantitation can be achieved by employing label-free approaches (Old et al., 2005; Neilson et al., 2011), chemical labeling 
such as isobaric tags (iTRAQ, TMT) (Thompson et al., 2003; Ong and Mann, 2005) or dynamic incorporation of stable isotopes into proteins based on metabolic activity (Ong et al., 2003; Beynon and Pratt, 2005; Jehmlich et al., 2008b). The incorporation of heavy isotopes, for example, ${ }^{13} \mathrm{C}$, in proteins can be detected on peptide level using a proteinstable isotope probing (protein-SIP) approach (Jehmlich et al., 2008b) and poses an additional way to unravel functionalities in relation to some specific processes such as hydrocarbon metabolism or degradation of organic xenobiotics. Diverse quantification methods were previously performed for pure cultures, but only label-free and metabolic labeling approaches were adopted for metaproteomic studies, and thus we will focus on them in the following sections.

\section{Label-free approaches}

Label-free quantitation has been frequently used to study natural communities and reliable results depend on standardized and reproducible liquid chromatography (LC) conditions (Neilson et al., 2011). Quantitation was performed using either signal intensity (area under the curve, AUC) or the number of fragmentation spectra (spectral counts, SC) (for review, see Neilson et al. (2011)). The AUC method is based on the detection of peptide ion abundances at specific retention times within given detection limits (Podwojski et al., 2010). In the SC quantitation, the numbers of identified fragment ion spectra per protein are counted, and calculation is based on the assumption that the number of fragment spectra for a peptide correlates with its abundance (Liu et al., 2004). This approach may lead to an overestimation of proteins of high molecular weight and peptide intensities. However, SC is recently becoming a common method for quantitative metaproteomic studies, as it is easy to implement for one-dimensional and two-dimensional proteomic data sets: Delmotte et al. (2009) used SC to roughly estimate protein abundances of 2257 proteins from phyllosphere microbiota, and Morris et al. (2010) evaluated the abundance of 2273 membrane proteins from microbial and viral communities of South Atlantic surface waters. The quantitation allowed them to clearly allocate molecular functions to specific phylotypes, which would not have been possible based on solely metagenome data. An improved SC approach is the normalized spectral abundance factor (NSAF), which considers the length of the quantified protein (Zybailov et al., 2007). The NSAF was used to analyze an acidophilic biofilm describing specific protein functions and ecological behaviors (Denef et al., 2010; Mueller et al., 2010; Jiao et al., 2011). Jiao et al. (2011) revealed that the abundance of 20 proteins account for more then the half of the 1351 identified proteins. The relative abundance of 6296 proteins from 28 biofilm samples were analyzed by hierarchical clustering and nonmetric multi dimensional scaling (Mueller et al., 2010). The NSAF of proteins within large multivariate data sets were compared and changes in the proteome of high and low abundant bacteria were discussed regarding the inhabiting biogeochemical zones. Microbial populations within distinct layers of a lake were investigated by Lauro et al. (2011) to compare 1824 proteins regarding their spatial distribution, and Schneider et al. (2012) studied the functionalities and microbial dynamics of litter degradation. Again, quantitation was the key for understanding the contribution of community members to the observed systemic activity. Recently, NSAF values were used to compare the effectiveness of different methods for the extraction of soil proteins (Keiblinger et al., 2012). Signal intensity measurements or AUC have been rarely used in metaproteomic studies, as they require highly reproducible chromatographic runs and are not very amenable to highly fractionated samples before LC-MS/MS (Neilson et al., 2011). A metaproteomic study described the biostimulation of an uranium-reducing aquifer community (Callister et al., 2010). They detected the shift of protein abundances by the AUC method in microbial consortia originating from different groundwater wells temporarily stimulated with a supply of acetate and were capable to allocate them to selected species. The effect of biostimulation of naphthalenedegrading enrichment cultures was described by Guazzaroni et al. (2013). Protein abundances of the cultures with or without a prior biostimulation showed a broad metabolic activity of the first one and specialized naphthalene degradation potential for the latter.

In the recent years, the identification and quantification of proteins in the gut microbiota became a hot topic in microbial ecology. The human gut microbiota was investigated starting by a 2-DE study (Klaassens et al., 2007), where a time-resolved analysis of protein spot changes was applied. Further on, gut microbiota studies were performed using 1-DE or label-free proteomic approaches. At first, Verberkmoes et al. (2009b) published an analysis employing relative protein abundances (normalized spectral) for a comparison of two fecal samples and two distinct sequence databases. Cantarel et al. (2011) used the same samples to test several computational strategies to improve protein identification. A new iterative workflow for protein identification leads to an increase of Clusters of Orthologous Groups (COG) categories (Rooijers et al., 2011). Special emphasis has been placed on the role of the gut microbiota for metabolic syndromes, inflammation and obesity. Therefore, feces of obese and lean adolescents were comparatively investigated with the results that a core proteome of 505 proteins with equal abundance was identified (counted by AUC) (Ferrer et al., 2013). Differences in the gene and protein composition between healthy 
and Crohn's disease twin pairs were studied by metagenomic and metaproteomic analysis (Erickson et al., 2012). Besides protein identification and quantitation, clear differences between noninflammation and inflammation in the ileum were observed. Furthermore, the alteration of the microbiota during an antibiotic treatment was studied in a multi-omics approach (Peréz-Cobas et al., 2012). Besides metagenomics, metatranscriptomics and metametabolomics, a comprehensive analysis of the metaproteome was performed. Microbial populations showed oscillatory changes during the therapy, indicating an alteration of the gut microbiota and the strong interaction with the host. Feces analyses revealed only limited insight to the interface between host and microbiota. Thus, two studies focussed on the mucosa-associated microbiota in human and rats (Li et al., 2011; Haange et al., 2012). Representative, Haange et al. (2012) showed phylogenetic and functional differences along the intestinal tract (mucosa and gut content) of rats. The above-mentioned studies showed that metaproteomics goes clearly beyond mere identification because it allows functional classification and abundance correlation with consortia members.

\section{Metabolic labeling}

Besides label-free quantitation, protein abundances and expression patterns can be calculated by labeling strategies. Chemical labeling uses tags that are added after the cultivation; metabolic labeling uses the metabolic activity of the organisms to incorporate stable isotopes. In contrast to chemical labeling approaches, metabolic labeling techniques lead to the incorporation of the quantitation markers within the culture and thereby avoid artefacts from processing or chemical reactions. The most common approach for eukaryotes is stable isotope labeling with amino acids in cell culture (SILAC). There, isotope-labeled arginine and/or lysine are supplied and hence incorporated into the proteins during cultivation. After trypsin cleavage, they constitute the C-terminal amino acid and lead to at least one labeled amino acid per peptide. Combination of peptides and concomitant MS measurement allow the relative quantification of an unlabeled to a labeled sample in one measurement, hence avoiding any variability based on slight day-to-day differences in LC-MS/MS analysis. Despite being usually applied to organisms auxotrophic for arginine/ lysine, the technique has also been used successfully for nematodes (Larance et al., 2011) and bacteria such as Staphylococcus aureus (Schmidt et al., 2010), which, although not being auxotrophic, were opportunistic enough to prefer the supplemented amino acids for peptide biosynthesis. Instead of amino acids, isotopically labeled substrates or nutrients can be applied that lead to a defined relative isotope abundance (RIA) in the biomass.
Peptides derived from these experiments can then be mixed with a control and quantified on the basis of peak intensities of labeled and non-labeled peptide signals. This method has been described using ${ }^{15} \mathrm{~N}$ (Snijders et al., 2005; Pan et al., 2011) and also ${ }^{36} \mathrm{~S}$ (Jehmlich et al., 2012). The incorporation of stable isotopes into proteins by metabolic labeling can be used to quantify protein abundances and to observe changes in the microbial community structure. For static comparisons, ${ }^{15} \mathrm{~N}$ was used as an isotopic metabolic label to detect proteome changes of an acidophilic biofilm (Belnap et al., 2010, 2011; Mueller et al., 2011). The community of interest was grown in the presence of ${ }^{15} \mathrm{~N}$-labeled medium to enable a $>95 \%{ }^{15} \mathrm{~N}$ labeling of the proteins. It was first used to prove that laboratory cultures are comparable to natural samples and further to show a differentiation of the metaproteome after cultivation of the biofilm in a $\mathrm{pH}$ range of 0.85-1.45 (Belnap et al., 2010, 2011). The spatial and temporal succession of the biofilm was studied using the ${ }^{15} \mathrm{~N}$-labeling approach (Mueller et al., 2011). Beside the sole description of community structure, the analysis of functionalities allocated to the various members of the community is of particular interest. This requires more detailed information about isotope label distribution than accessible by mere quantitative metaproteomics. Protein-SIP is a method likely able to provide this additional information.

\section{Features of protein-SIP to simultaneously detect metabolic activity and phylogeny}

Protein-SIP is based on the metabolic incorporation of isotopically labeled substrates, where ${ }^{13} \mathrm{C},{ }^{15} \mathrm{~N}$ or ${ }^{36} \mathrm{~S}$ can be used (Jehmlich et al., 2008a, b, 2012). The metabolism of the labeled substrates leads to assimilation of heavy isotopes and subsequent incorporation into the various classes of biomolecules, including proteins and can be detected by mass spectrometry of peptides as reviewed in Seifert et al. (2012). The amount of atoms replaced by their heavy isotopes changes the natural isotope composition and is defined as the RIA. The RIA can be determined by LC-MS/MS analysis at the peptide level (Table 1). The calculation of the RIA is either done by analysis of the distribution of different isotope patterns of the peptides or based on features of the peptide mass such as the relation of the parent mass to the first two digits (Jehmlich et al., 2008b; Fetzer et al., 2010; Jehmlich et al., 2010; Taubert et al., 2011a). For the first approach, the amino-acid sequence of the peptide is needed to calculate the elemental composition and the theoretical mass, thus this approach requires suitable sequence information. The calculation of the RIA includes the whole isotope pattern, which allows a highly accurate calculation of the RIA; enabling differentiation of deviations down to $0.1 \%$ with only 10 peptides (Taubert et al., 2011a). The second 
Table 1 Numerical parameters (RIA, LR) and features (shape) of protein-SIP posing carbon and nitrogen fluxes in microbial communities.

$R I A$

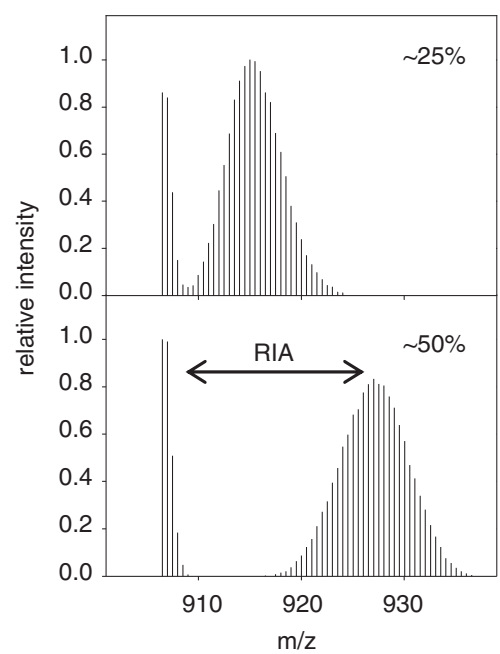

Percentage of stable isotope incorporation Calculated by the extent of the mass shift of the peptide peak

Reflects the carbon and nitrogen, respectively, metabolization

Limit of detection:

$1 \%{ }^{13} \mathrm{C}$ and ${ }^{15} \mathrm{~N}$

References:

Taubert et al., 2011a;

Taubert et al., 2013
$L R$

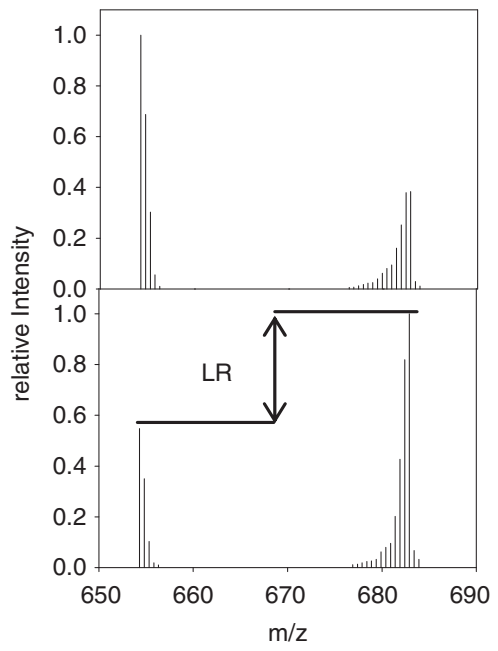

Ratio between the intensity of the isotope pattern of the labeled peptide and the total intensity of isotope patterns of unlabeled and labeled peptide

Reflects protein synthesis and turnover in time-resolved analysis

References:

Taubert et al., 2011b
Shape of isotope pattern

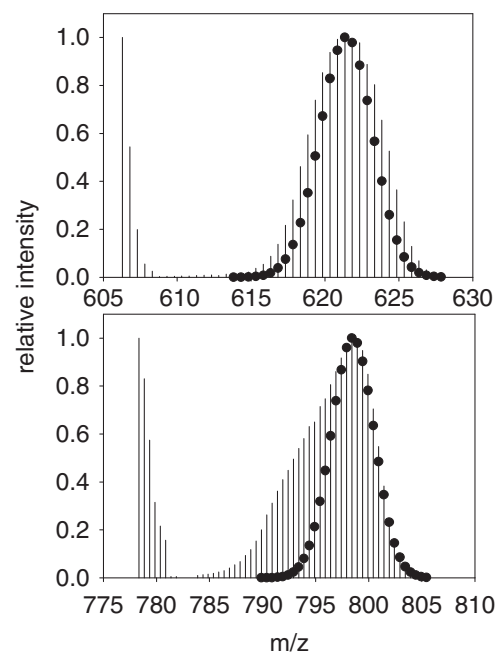

Incorporation of ${ }^{13} \mathrm{C}$ in proteins (<99\%) yields Gaussian distribution of the isotope patterns of a peptide

Ideal Gaussian distribution indicates

direct substrate utilization

Isotope pattern with negative skewness

indicates indirect ${ }^{13} \mathrm{C}$ substrate

metabolisation

References:

Morris et al., 2012;

Taubert et al., 2012

Abbreviations: LR, labeling ratio; protein-SIP, protein-based stable isotope probing; RIA, relative isotope abundance.

RIA: MS spectra of peptide GIFAVGDVATWPLHSGGK from the benzene 1,2-dioxygenase ferredoxin-NAD $(+)$ reductase subunit protein $(P$ putida) with a RIA of $\sim 25 \%$ and $\sim 50 \%$; LR: MS spectra of peptide EQAFVM(ox)NPEVK of elongation factor Ts at an early (above) and later time point (below); shape: peptide SDKIDIYSDR of methyl-coenzyme M reductase, beta subunit from M. thermophila with a RIA of 59\% (above), peptide LAENGMMFDMLQR of methyl-coenzyme M reductase, gamma subunit from M. marisnigri with a RIA of 59\% and clear left-tailing (below).

approach, called half decimal place rule, is sequence independent and the RIA calculation is based on the first two digits of the peptide mass. The major disadvantage of this method is the requirement of at least 40 peptides with equal RIA to achieve accuracy below 10\% deviation (Jehmlich et al., 2010). Besides the amount of heavy isotopes within the molecules, the ratio between labeled and unlabeled molecules also contains valuable information. The 'labeling ratio' (LR) is based on the intensity ratio of the respective peptide MS signals (Table 1). The assessment of the LR allows, for example, the quantitation of protein biosynthesis as shown in a time series study by Taubert et al. (2011b), where protein induction by substrate shift was analyzed. Another valuable feature of proteinSIP is the possibility to detect differences in substrate usage by analysis of the shapes of isotope patterns in peptides. Direct metabolisation of isotopically labeled substrates leads to an isotope pattern closely resembling a normal distribution
(Table 1), whereas cross-feeding, that is, usage of labeled intermediates from degradation processes accumulating in culture, result in tailed distributions. Hence, the incorporation of isotopes into proteins of different groups of microorganisms can be used to track carbon fluxes within the studied consortia. The corresponding phylogenetic resolution depends on the available (meta-)genome sequences, the diversity of the phyla and the detection of valuable phylogenetic marker proteins such as chaperonin, ATPases and ribosomal proteins (Taubert et al., 2012).

\section{Applications of protein-SIP in microbial ecology}

The suitability of protein-SIP to detect key metabolic players within a community was first shown using a defined nitrate-reducing artificial mixed culture of the toluene-degrading Aromatoleum aromaticum 

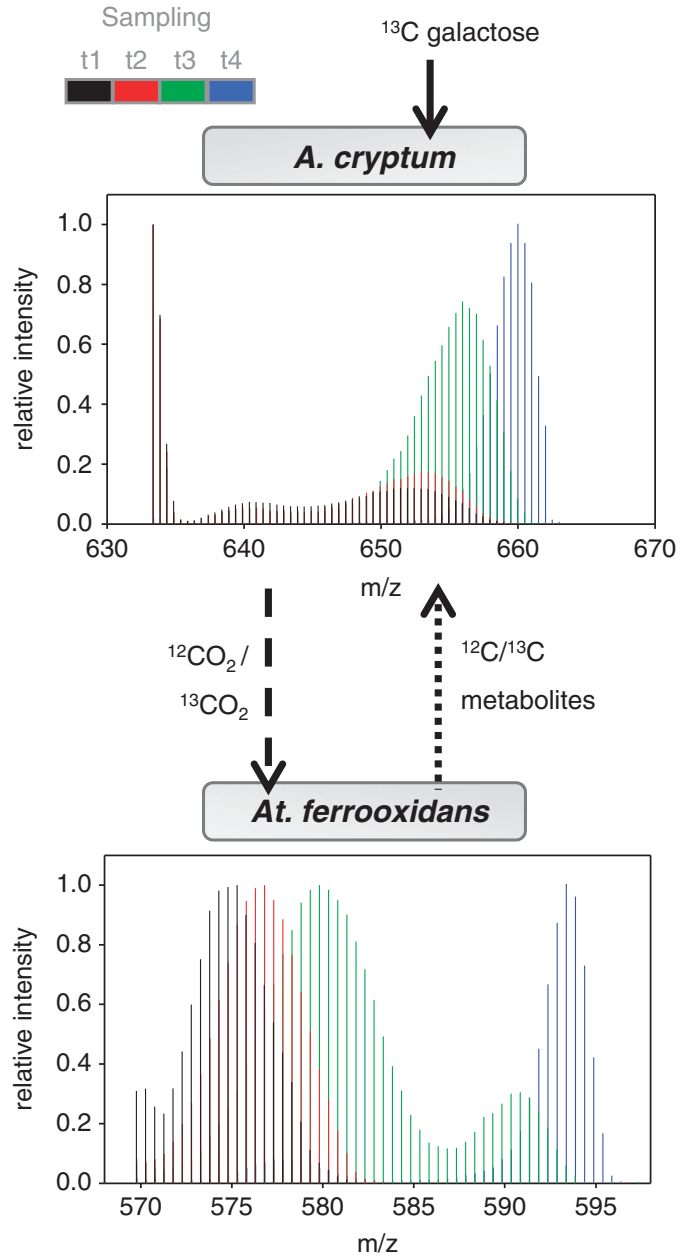

Figure 1 Overview of a protein-SIP experiment studying the carbon flux between heterotrophic (Acidiphilum cryptum) and autotrophic (Acidithiobacillus ferrooxidans) organisms over four sampling points (t1-t4) (Kermer et al., 2012). Labeled galactose is metabolized by $A$. cryptum to labeled $\mathrm{CO}_{2}$. In early time points, only a low amount of labeled $\mathrm{CO}_{2}$ is present and is fixed by the autotrophic strain, leading to a low RIA. In later time points, the metabolic activity of $A$. cryptum resulted in a strong increase in ${ }^{13} \mathrm{CO}_{2}$, leading to a higher RIA in $A$. ferrooxidans. The ${ }^{13} \mathrm{C}$-flux was reconstructed by the changes of the isotopologue patterns (color code, see sampling bar in the figure). Representative mass spectra showing the peptide ion mass distribution of peptide AGGLPAVIGELIR of dihydroxy-acid dehydratase (A. cryptum, gi148259108) and peptide AFDGSSIAGWK of glutamine synthetase, type I (A. ferrooxidans, gi198282766).

EbN1 and a gluconate-degrading enrichment culture not able to use toluene as substrate (Jehmlich et al., 2008b). The mixed culture was fed with ${ }^{13} \mathrm{C}$-labeled toluene and ${ }^{12} \mathrm{C}$-gluconate as carbon sources. Although all members of the consortium grew under these conditions, ${ }^{13} \mathrm{C}$-labeled proteins were exclusively assigned to strain EbN1, demonstrating the suitability of protein-SIP to detect and identify ${ }^{13} \mathrm{C}$ labeled proteins in a mixed culture. Protein-SIP was further established as a suitable method to trace the carbon flux between a heterotrophic and an autotrophic bacterial species by Kermer et al. (2012). In the course of the labeling experiments, the development of the RIA and the LR of peptides revealed that a carbon transfer occurred when $\mathrm{CO}_{2}$ formed by heterotrophic metabolism was used by the autotrophic species as a carbon source (Figure 1). The mass shifts of the peptides caused by the alternating incorporation of ${ }^{13} \mathrm{C}$ are depicted as colored isotopologue patterns in Figure 1. Protein-SIP applications in more complex cultures and environmental samples were conducted first in a study on a MTBEdegrading culture (Bastida et al., 2010). The microbial community structure was analyzed after growth on ${ }^{13} \mathrm{C}$-MTBE revealing that proteins related to members of the Comamonadaceae family were not ${ }^{13} \mathrm{C}$-labeled, whereas proteins related to Methylibium petroleiphilum PM1 showed an average ${ }^{13} \mathrm{C}$ RIA of 94.5 atom $\%{ }^{13} \mathrm{C}$. In this study, the phylogenetic information retrieved from proteins correlated well with the structure of the microbial community established by $16 \mathrm{~S}$ rRNA gene libraries. In further studies, a relationship between RNA-SIP and protein-SIP data was published by Jechalke et al. (2013). The active fraction of an aerobic benzenedegrading microbial community was identified as members of Beta- and Gammaproteobacteria with RIAs of about 90 atom $\%{ }^{13} \mathrm{C}$. The detection of ${ }^{13} \mathrm{C}$-labeled proteins related to toluene dioxygenase and catechol 2,3-dioxygenase suggested benzene degradation by a dihydroxylation pathway with subsequent meta-cleavage of the catechol formed. Recently, protein-SIP was used to characterize the functionality of a sulfate-reducing community with $m$-xylene as the sole source of carbon and energy (Bozinovski et al., 2012). Out of the 331 identified proteins, $23 \%$ were not ${ }^{13} \mathrm{C}$-labeled, whereas $77 \%$ showed an incorporation of $19-22$ atom $\%{ }^{13} \mathrm{C}$. ${ }^{13} \mathrm{C}$-labeled proteins were involved in anaerobic $m$-xylene biodegradation, in sulfate reduction, in the Wood-Ljungdahl pathway and in general housekeeping processes. Thirty-eight percent of the labeled proteins were affiliated to Deltaproteobacteria, which suggests a key role of this group in the assimilation of $m$-xylene. Morris et al. (2012) studied the carbon flow in an anaerobic hydrocarbon degrading consortium. The methanogenic culture was incubated either with an oil-containing or an oil-free solid matrix and amended with either ${ }^{13} \mathrm{C}$-labeled $n$-fatty acid or $n$-alkane. Proteins were identified with an in silicocreated metagenome consisting of public genomes of sulfate-reducing species, fatty acid-metabolizing species and methanogenic archaea. Beside yielding different ${ }^{13} \mathrm{C}$ RIAs of the metabolic groups, depending on the used substrate and solid matrix, protein-SIP allowed a differentiation of direct and indirect carbon utilization based on peptides' isotope patterns (Table 1). The authors posed that peptides belonging to Methanoculleus marisnigri, which metabolizes $\mathrm{CO}_{2}$ and other $\mathrm{C} 1$ substrates, showed patterns typical for consumption of degradation products of a labeled substrate, while peptides from Methanosaeta thermophila, an acetoclastic methanogen, showed distributions resembling a direct usage of the labeled substrates. The results indicated that methanogenesis 

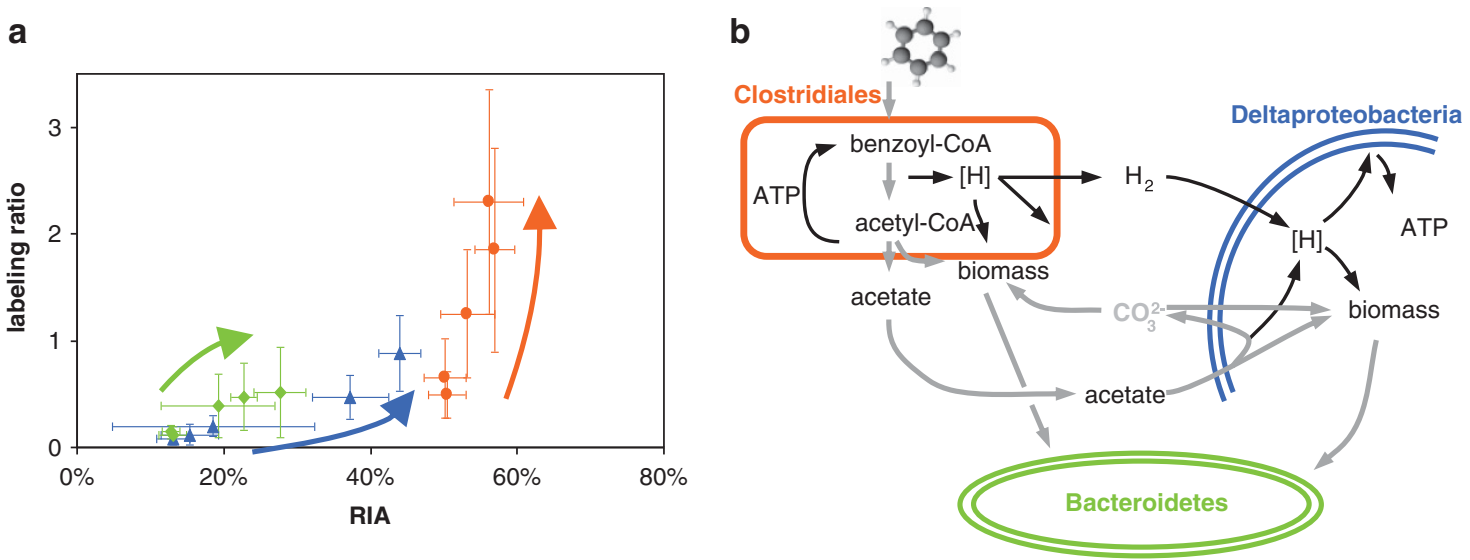

Figure 2 Data evaluation of a protein-SIP experiment to detect the carbon flux in an anaerobic benzene-degrading community (Taubert et al., 2012). (a) Protein grouping based on RIA vs LR to define metabolic groups of bacterial organisms obtained by ${ }^{13} \mathrm{C}$ benzene metabolisation. Colored data points indicate different metabolic groups: group 1, Clostridiales (orange dots); group 2, Deltaproteobacteria (blue triangles); group 3, Bacteroidetes (green squares). Arrows indicate the chronological development between the RIA and LR. A low variability of the RIA and a strong increase in LR (as shown for group 1) indicates a direct metabolisation of the ${ }^{13} \mathrm{C}$-labeled substrate and a high growth rate. A stronger change of RIA over time indicates an indirect metabolisation (like shown for group 2 and 3). (b) Model of the carbon flux with the bacterial community based on data of the protein-SIP experiment using ${ }^{13} \mathrm{C}$-labeled benzene and ${ }^{13} \mathrm{C}$-labeled carbonate.

in this consortium was driven by acetate metabolisation and only secondary by hydrogenotrophic $\mathrm{CO}_{2}$ reduction. The carbon flow in a sulfate-reducing, benzene-degrading community was recently studied (Taubert et al., 2012). The anaerobic aquifer community was set up either using ${ }^{13} \mathrm{C}$-labeled benzene and ${ }^{12} \mathrm{C}$ carbonate or ${ }^{12} \mathrm{C}$ benzene and ${ }^{13} \mathrm{C}$-labeled carbonate. Up to five samples were investigated over 300 days per experiment. According to the development of the RIA and the LR over time, three distinct groups of proteins could be defined, based on MS identifications from metagenomic sequences, thus underlining the importance of following a time course in proteinSIP experiments (Figure 2). Phylogenetic affiliation was performed by BLASTP-based annotation of the respective metagenome contigs. The metagenome was employed to identify proteins and the labeling experiment resulted in a reconstruction of the carbon flow in the microbial community. Species belonging to Clostridiales were defined as the primary benzene consumers and as heterotrophic $\mathrm{CO}_{2}$-fixing bacteria. ${ }^{13} \mathrm{C}$-labeled $\mathrm{CO}_{2}$ was also fixed by Deltaproteobacteria members and additionally they used ${ }^{13} \mathrm{C}$-labeled metabolites released by the Clostridiales. ${ }^{13} \mathrm{C}$-labeled secondary metabolites or dead biomass of both groups were probably used as the carbon sources of putative scavengers belonging to Bacteroidetes/ Chlorobi organisms defined as the third metabolic group in the consortium (Figure 2b).

Overall, protein-SIP is capable of providing insights into microbial consortia beyond the mere identification and quantification of proteins. Protein-SIP can (i) accurately detect metabolic activity and thereby distinguish between key players and bystanders in a microbial community, (ii) differentiate between the type of substrate usage and (iii) deliver information on protein induction. Further applications of protein-SIP may allow the deciphering of the main pathways and key players involved in the biodegradation of newly appearing pollutants, in the global carbon and nitrogen cycling in aquatic and terrestrial ecosystems and in the interaction between human and their microbiota.

\section{Future development: automatic calculation of isotope labeling}

To increase the throughput of mass spectra analysis for protein-SIP, an automated calculation of RIA and LR is needed. Currently, one automated pipeline is available and its application has been shown for ${ }^{15} \mathrm{~N}$-labeled proteins by transforming the whole in silico mass spectra from the sample-specific metagenome with increments of $1 \%$ in ${ }^{15} \mathrm{~N}$ RIA to identify species-specific migration patterns from established into regrowing communities of an acidmine drainage biofilm (Pan et al., 2011). Because of the stepwise transformation of a huge data set, the process required a relatively high amount of processing time in respect to more complex samples. In addition, a higher resolution with RIAs of $0.1 \%$ would even need more computational effort using the described bioinformatic pipeline. One alternative that has to be developed could be an automated tool, which identifies ${ }^{13} \mathrm{C}$ - or ${ }^{15} \mathrm{~N}$-labeled masses in correlation to the unlabeled ones within a certain retention time window and determines peptide sequences, RIAs and LRs in one step.

\section{Integration of protein-SIP in the toolbox of microbial ecology and conclusion}

The toolbox of microbial ecology comprises techniques that either use DNA, RNA, proteins and 


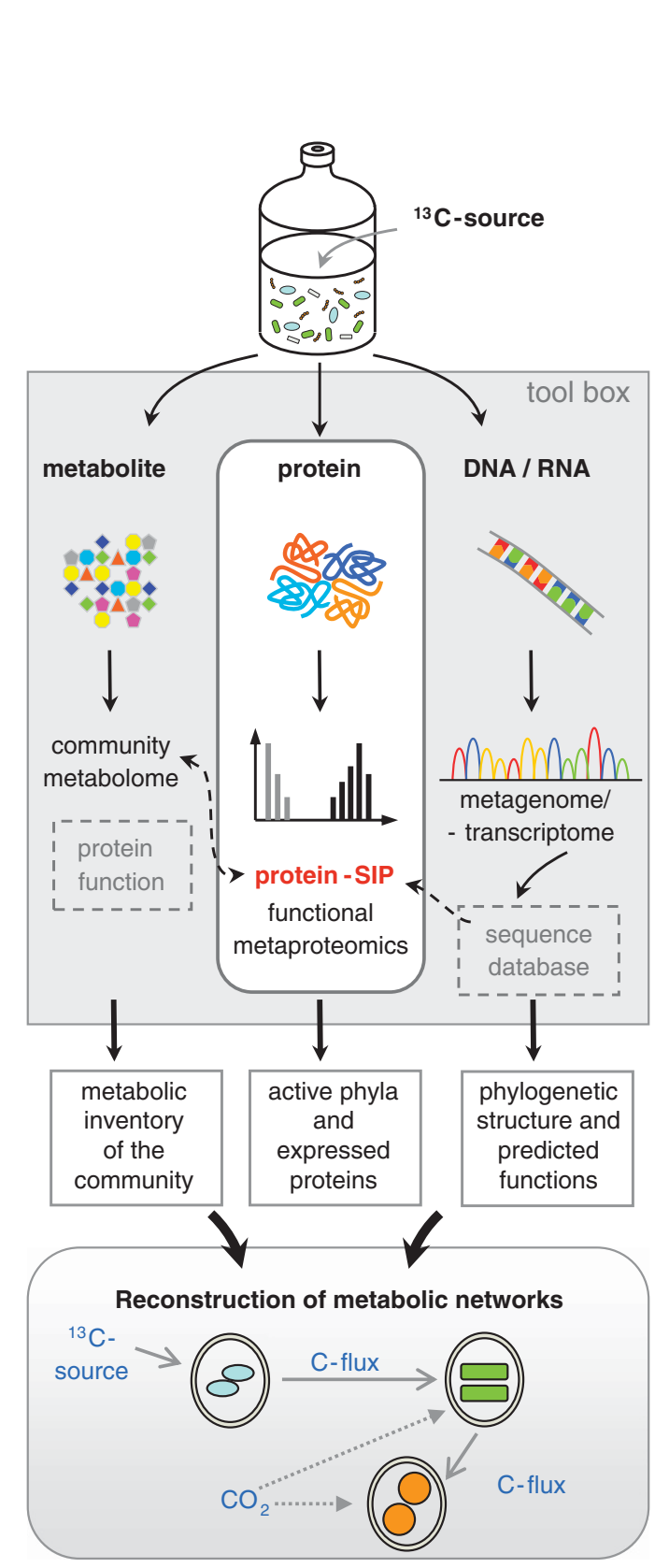

Figure 3 Integration of protein-SIP in the toolbox of microbial ecology and the combination of OMICS to reconstruct metabolic networks.

metabolites extracted from the whole community, or analyze intact single cells. The combination of SIP with these techniques leads to a holistic view into the metabolic networks (Figure 3). Single-cell analysis with nano secondary ion mass spectrometry is the most sensitive method to detect stable isotope incorporation. However, the gain of information about the phylogeny and function of the single cells depends highly on the used probes, and high-throughput experiments are not applicable due to the required machine time (Musat et al., 2012). This problem might have been solved by the development of Chip-SIP (Mayali et al., 2012). Raman microspectroscopy has a high spatial resolution but requires a label of at least 10 atom $\%$ for spectral shifts for identification of isotope enrichment in labeling experiments (Huang et al., 2007).

Within the SIP toolbox, protein-SIP is integrated as an interface between the nucleic acids-based techniques that provide the sequence database for MS analysis and the analysis of metabolites that indicate protein activities (Figure 3). In comparison to other SIP approaches three important features of protein-SIP are noteworthy: (i) minimal required incorporation of heavy isotope (about $0.1 \%$ of additional ${ }^{13} \mathrm{C}$ incorporation) compared with DNA/ RNA-SIP (50/10 atom\% (Manefield et al., 2002; Radajewski et al., 2003)), (ii) dynamic range for quantification of isotope composition (two orders of magnitude) and (iii) high resolution of species and function (depending on the database) due to the fact that proteomic studies are not dependent on the amplification of target genes. The combination of multi-omics approaches to quantify metabolic groups and by using meta-metabolome-SIP would lead into a comprehensive tool to track the nutrient fluxes within microbial communities.

\section{Conflict of Interest}

The authors declare no conflict of interest.

\section{Acknowledgements}

We acknowledge the financial support provided by the European Community Project MAGICPAH (FP7-KBBE2009-245226), the Deutsche Forschungsgemeinschaft (SPP1319, FOR 1530), BMBF IFB Adiposity Disease and by the Helmholtz Impulse and Networking Fund through Helmholtz Interdisciplinary Graduate School for Environmental Research (HIGRADE).

\section{References}

Bastida F, Rosell M, Franchini AG, Seifert J, Finsterbusch S, Jehmlich N et al. (2010). Elucidating MTBE degradation in a mixed consortium using a multidisciplinary approach. FEMS Microbiol Ecol 73: 370-384.

Belnap CP, Pan C, Denef VJ, Samatova NF, Hettich RL, Banfield JF. (2011). Quantitative proteomic analyses of the response of acidophilic microbial communities to different pH conditions. ISMEJ 5: 1152-1161.

Belnap CP, Pan C, VerBerkmoes NC, Power ME, Samatova NF, Carver RL et al. (2010). Cultivation and quantitative proteomic analyses of acidophilic microbial communities. ISMEJ 4: 520-530.

Beynon RJ, Pratt JM. (2005). Metabolic labeling of proteins for proteomics. Mol Cell Proteomics 4: 857-872.

Bozinovski D, Herrmann S, Richnow HH, von Bergen M, Seifert J, Vogt C. (2012). Functional analysis of an anaerobic $m$-xylene-degrading enrichment culture using protein-based stable isotope probing. FEMS Microbiol Ecol 81: 134-144.

Callister SJ, Wilkins MJ, Nicora CD, Williams KH, Banfield JF, Verberkmoes NC et al. (2010). Analysis of biostimulated microbial communities from two field experiments reveals temporal and spatial differences in proteome profiles. Environ Sci Technol 44: 8897-8903. 
Cantarel BL, Erickson AR, VerBerkmoes NC, Erickson BK, Carey PA, Pan C et al. (2011). Strategies for metagenomic-guided whole-community proteomics of complex microbial environments. PLOS ONE 6: e27173.

Delmotte N, Knief C, Chaffron S, Innerebner G, Roschitzki $\mathrm{B}$, Schlapbach $\mathrm{R}$ et al. (2009). Community proteogenomics reveals insights into the physiology of phyllosphere bacteria. Proc Natl Acad Sci USA 106: 16428-16433.

Denef VJ, Kalnejais LH, Mueller RS, Wilmes P, Baker BJ, Thomas BC et al. (2010). Proteogenomic basis for ecological divergence of closely related bacteria in natural acidophilic microbial communities. Proc Natl Acad Sci USA 107: 2383-2390.

Erickson AR, Cantarel BL, Lamendella R, Darzi Y, Mongodin EF, Pan C et al. (2012). Integrated metagenomics/metaproteomics reveals human host-microbiota signatures of Crohn's disease. PLOS ONE 7: e49138.

Ferrer M, Ruiz A, Lanza F, Haange SB, Oberbach A, Till H et al. (2013). Microbiota from the distal guts of lean and obese adolescents exhibit partial functional redundancy besides clear differences in community structure. Environ Microbiol 15: 211-226.

Fetzer I, Jehmlich N, Vogt C, Richnow HH, Seifert J, Harms $\mathrm{H}$ et al. (2010). Calculation of partial isotope incorporation into peptides measured by mass spectrometry. BMC Res Notes 3: 178.

Guazzaroni ME, Herbst FA, Lores I, Tamames J, Pelaez AI, Lopez-Cortes N et al. (2013). Metaproteogenomic insights beyond bacterial response to naphthalene exposure and bio-stimulation. ISMEJ 7: 122-136.

Haange SB, Oberbach A, Schlichting N, Hugenholtz F, Smidt H, von Bergen M et al. (2012). Metaproteome analysis and molecular genetics of rat intestinal microbiota reveals section and localization resolved species distribution and enzymatic functionalities. J Proteome Res 11: 5406-5417.

Huang WE, Stoecker K, Griffiths R, Newbold L, Daims H, Whiteley AS et al. (2007). Raman-FISH: combining stable-isotope Raman spectroscopy and fluorescence in situ hybridization for the single cell analysis of identity and function. Environ Microbiol 9: 1878-1889.

Jechalke S, Franchini AG, Bastida F, Bombach P, Rosell M, Seifert J et al. (2013). Analysis of structure, function and activity of a benzene degrading microbial community. FEMS Microbiol Ecol (doi:10.1111/1574-6941. 12090).

Jehmlich N, Fetzer I, Seifert J, Mattow J, Vogt C, Harms H et al. (2010). Decimal place slope, a fast and precise method for quantifying ${ }^{13} \mathrm{C}$ incorporation levels for detecting the metabolic activity of microbial species. Mol Cell Proteomics 9: 1221-1227.

Jehmlich N, Kopinke FD, Lenhard S, Herbst FA, Seifert J, Lissner U et al. (2012). Sulphur- ${ }^{36} \mathrm{~S}$ stable isotope labeling of amino acids for quantification (SULAQ). Proteomics 12: 37-42.

Jehmlich N, Schmidt F, Hartwich M, von Bergen M, Richnow HH, Vogt C. (2008a). Incorporation of carbon and nitrogen atoms into proteins measured by proteinbased stable isotope probing (Protein-SIP). Rapid Commun Mass Spectrom 22: 2889-2897.

Jehmlich N, Schmidt F, von Bergen M, Richnow HH, Vogt C. $(2008 \mathrm{~b})$. Protein-based stable isotope probing (Protein-SIP) reveals active species within anoxic mixed cultures. ISMEJ 2: 1122-1133.
Jiao Y, D’Haeseleer P, Dill BD, Shah M, Verberkmoes NC, Hettich RL et al. (2011). Identification of biofilm matrix-associated proteins from an Acid mine drainage microbial community. Appl Environ Microbiol 77: 5230-5237.

Keiblinger KM, Wilharititz IC, Schneider T, Roschitzki B, Schmid E, Eberl L et al. (2012). Soil metaproteomicsComparative evaluation of protein extraction protocols. Soil Biol Biochem 54: 14-24.

Keller M, Hettich R. (2009). Environmental proteomics: a paradigm shift in characterizing microbial activities at the molecular level. Microbiol Mol Biol Rev 73: 62-70.

Kermer R, Hedrich S, Taubert M, Baumann S, Schlömann M, Johnson DB et al. (2012). Elucidation of carbon transfer in a mixed culture of Acidiphilium cryptum and Acidithiobacillus ferrooxidans using proteinbased stable isotope probing. JIOMICS 2: 37-45.

Klaassens ES, de Vos WM, Vaughan EE. (2007). Metaproteomics approach to study the functionality of the microbiota in the human infant gastrointestinal tract. Appl Environ Microbiol 73: 1388-1392.

Larance M, Bailly AP, Pourkarimi E, Hay RT, Buchanan G, Coulthurst $S$ et al. (2011). Stable-isotope labeling with amino acids in nematodes. Nat Methods 8: 849-851.

Lauro FM, DeMaere MZ, Yau S, Brown MV, Ng C, Wilkins D et al. (2011). An integrative study of a meromictic lake ecosystem in Antarctica. ISMEJ 5: 879-895.

Li X, LeBlanc J, Truong A, Vuthoori R, Chen SS, Lustgarten JL et al. (2011). A metaproteomic approach to study human-microbial ecosystems at the mucosal luminal interface. PLoS ONE 6: e26542.

Liu H, Sadygov RG, Yates JR 3rd. (2004). A model for random sampling and estimation of relative protein abundance in shotgun proteomics. Anal Chem 76: 4193-41201.

Manefield M, Whiteley AS, Ostle N, Ineson P, Bailey MJ. (2002). Technical considerations for RNA-based stable isotope probing: an approach to associating microbial diversity with microbial community function. Rapid Commun Mass Spectrom 16: 2179-2183.

Mayali X, Weber PK, Brodie EL, Mabery S, Hoeprich PD, Pett-Ridge J. (2012). High-throughput isotopic analysis of RNA microarrays to quantify microbial resource use. ISMEJ 6: 1210-1221.

Morris BEL, Herbst FA, Bastida F, Seifert J, von Bergen M, Richnow $\mathrm{HH}$ et al. (2012). Microbial interactions during residual oil and n-fatty acid metabolism by a methanogenic consortium. Environ Microbiol Rep 4: 297-306.

Morris RM, Nunn BL, Frazar C, Goodlett DR, Ting YS, Rocap G. (2010). Comparative metaproteomics reveals ocean-scale shifts in microbial nutrient utilization and energy transduction. ISMEJ 4: 673-685.

Mueller RS, Denef VJ, Kalnejais LH, Suttle KB, Thomas BC, Wilmes $\mathrm{P}$ et al. (2010). Ecological distribution and population physiology defined by proteomics in a natural microbial community. Mol Syst Biol 6: 374.

Mueller RS, Dill BD, Pan C, Belnap CP, Thomas BC, Verberkmoes NC et al. (2011). Proteome changes in the initial bacterial colonist during ecological succession in an acid mine drainage biofilm community. Environ Microbiol 13: 2279-2292.

Musat N, Foster R, Vagner T, Adam B, Kuypers MMM. (2012). Detecting metabolic activities in single cells, with emphasis on nanoSIMS. FEMS Microbiol Rev 36: 486-511. 
Neilson KA, Ali NA, Muralidharan S, Mirzaei M, Mariani M, Assadourian G et al. (2011). Less label, more free: approaches in label-free quantitative mass spectrometry. Proteomics 11: 535-553.

Old WM, Meyer-Arendt K, Aveline-Wolf L, Pierce KG, Mendoza A, Sevinsky JR et al. (2005). Comparison of label-free methods for quantifying human proteins by shotgun proteomics. Mol Cell Proteomics 4: 1487-1502.

Ong SE, Foster LJ, Mann M. (2003). Mass spectrometricbased approaches in quantitative proteomics. Methods 29: $124-130$.

Ong SE, Mann M. (2005). Mass spectrometry-based proteomics turns quantitative. Nat Chem Biol 1: 252-262.

Pan C, Fischer CR, Hyatt D, Bowen BP, Hettich RL, Banfield JF. (2011). Quantitative tracking of isotope flows in proteomes of microbial communities. Mol Cell Proteomics 10: 006049.

Peréz-Cobas AE, Gosalbes MJ, Friedrichs A, Knecht H, Artacho A, Eismann K et al. (2012). Gut microbiota disturbance during antibiotic therapy: a multiomic approach. Gut, Doi:10.1136/gutjnl-2012-303184 (in press).

Podwojski K, Eisenacher M, Kohl M, Turewicz M, Meyer HE, Rahnenfuhrer J et al. (2010). Peek a peak: a glance at statistics for quantitative label-free proteomics. Expert Rev Proteomics 7: 249-261.

Radajewski S, McDonald IR, Murrell JC. (2003). Stableisotope probing of nucleic acids: a window to the function of uncultured microorganisms. Curr Opin Biotechnol 14: 296-302.

Rooijers K, Kolmeder C, Juste C, Dore J, de Been M, Boeren $S$ et al. (2011). An iterative workflow for mining the human intestinal metaproteome. BMC Genom 12: 6.

Schmidt F, Scharf SS, Hildebrandt P, Burian M, Bernhardt J, Dhople V et al. (2010). Time-resolved quantitative proteome profiling of host-pathogen interactions: the response of Staphylococcus aureus RN1HG to internalisation by human airway epithelial cells. Proteomics 10: 2801-2811.

Schneider T, Keiblinger KM, Schmid E, SterflingerGleixner K, Ellersdorfer G, Roschitzki B et al. (2012). Who is who in litter decomposition? Metaproteomics reveals major microbial players and their biogeochemical functions. ISMEJ 6: 1749-1762.

Schneider T, Riedel K. (2010). Environmental proteomics: analysis of structure and function of microbial communities. Proteomics 10: 785-798.
Seifert J, Taubert M, Jehmlich N, Schmidt F, Volker U, Vogt C et al. (2012). Protein-based stable isotope probing (protein-SIP) in functional metaproteomics. Mass Spectrom Rev 31: 683-697.

Snijders APL, de Koning B, Wright PC. (2005). Perturbation and interpretation of nitrogen isotope distribution patterns in proteomics. J Proteome Res 4: 2185-2191.

Taubert M, Baumann S, von Bergen M, Seifert J. (2011a). Exploring the limits of robust detection of incorporation of ${ }^{13} \mathrm{C}$ by mass spectrometry in protein-based stable isotope probing (protein-SIP). Anal Bioanal Chem 401: 1975-1982.

Taubert M, Jehmlich N, Vogt C, Richnow HH, Schmidt F, von Bergen $\mathrm{M}$ et al. (2011b). Time resolved protein-based stable isotope probing (Protein-SIP) analysis allows quantification of induced proteins in substrate shift experiments. Proteomics 11: 2265-2274.

Taubert M, Vogt C, Wubet T, Kleinsteuber S, Tarkka MT, Harms H et al. (2012). Protein-SIP enables time-resolved analysis of the carbon flux in a sulfate-reducing, benzene-degrading microbial consortium. ISMEJ 6: 2291-2301.

Taubert M, von Bergen M, Seifert J. (2013). Limitations in detection of ${ }^{15} \mathrm{~N}$ incorporation by mass spectrometry in protein-based stable isotope probing (protein-SIP). Anal Bioanal Chem 405: 3989-3996.

Thompson A, Schafer J, Kuhn K, Kienle S, Schwarz J, Schmidt G et al. (2003). Tandem mass tags: a novel quantification strategy for comparative analysis of complex protein mixtures by MS/MS. Anal Chem 75: 1895-1904.

Verberkmoes NC, Denef VJ, Hettich RL, Banfield JF. (2009a). Systems biology: Functional analysis of natural microbial consortia using community proteomics. Nature Rev Microbiol 7: 196-205.

Verberkmoes NC, Russell AL, Shah M, Godzik A, Rosenquist M, Halfvarson J et al. (2009b). Shotgun metaproteomics of the human distal gut microbiota. ISMEJ 3: 179-189.

Wilmes P, Bond PL. (2009). Microbial community proteomics: elucidating the catalysts and metabolic mechanisms that drive the Earth's biogeochemical cycles. Curr Opin Microbiol 12: 310-317.

Zybailov BL, Florens L, Washburn MP. (2007). Quantitative shotgun proteomics using a protease with broad specificity and normalized spectral abundance factors. Mol Biosyst 3: 354-360. 